\title{
OUT-OF-PLANE STATIC CRUSHING OF THIN-WALLED HONEYCOMB STRUCTURES. NUMERICAL AND EXPERIMENTAL INVESTIGATION
}

\author{
Radosław Ciepielewski', Paweł Dybcio ${ }^{1}$, Wiesław Barnat ${ }^{1}$
}
1 Faculty of Mechanical Engineering, Department of Mechanics and Applied Computer Science, Military University of Technology, Kaliskiego 2 st., 00-908 Warsaw, Poland, e-mail: radoslaw.ciepielewski@wat.edu.pl, pawel.dybcio@wat.edu.pl

Received: 2018.05.18

Accepted: 2018.08.01 Published: 2018.09.01

\begin{abstract}
Aim of presented study was to compare plateau stress in honeycomb structures under out-of-plane load calculated using Wierzbicki formula with numerical simulations validated using experimental trials. ALUBOND ${ }^{\circledR}$ Alucore honeycomb structure was examined. The results of theoretical, experimental and numerical investigation are reported. Two methods of modeling core behavior were evaluated using simulations. Full core geometry and simplified Y-shaped element were analyzed. Both approaches were compared with experimental out-of-plane compression tests. Aim of the study was to determine the influence of core geometrical parameters on obtained plateau stress value. Various foil thicknesses and cell sizes were studied numerically. The results showed, that initial and final deformation mode strongly depends on the geometry of the honeycomb structure. Force required to crush the core grew with increase of wall thickness, and decreased with increase of cell size. Calculations were performed using an implicit integration scheme implemented in the LS-DYNA software. Research showed the presence of plateau relationship between stress and geometric dimensions and structure response. Good agreement between results obtained by all methods was achieved. Basing on the results, conclusions concerning modeling honeycomb materials were drawn.
\end{abstract}

Keywords: honeycomb, thin-walled structure, static compression test, out-of-plane compression, FEM, LS-DYNA, Alucore.

\section{INTRODUCTION}

Sandwich structures are composed of a thick lightweight core bonded by adhesion to two thin and stiff skins. Many types of lightweight cores are known. One of them, thin-walled cellular structure, is called honeycomb. McFarland [10] reported results of experimental investigation on different types of thin-walled energy-absorbing structures. Research showed, that honeycomb structures have the best relation of mechanical properties to homogenous density comparing to other thin-walled structures.

Figure 1 presents comparison between energy absorption in out-of-plane (red) and in two in plane directions (blue \& green) reported by Ivañez et al. [6]. Honeycomb structures have orthotropic mechanical properties. They have much more outof-plane stiffness than in any other direction. It was reported by many researchers $[1,6,12]$.

Paik [7] studied nature of honeycomb crushing force under different static load cases. In his study, the ultimate and plateau force values in relation to core density characteristics were determined. As well as that, honeycomb cores have good energy-absorbing capabilities in compression, as reported in Gibson and Asby study [4]. They also concluded, that in order to obtain energy-absorbing properties of structure, axial compression tests are needed. 


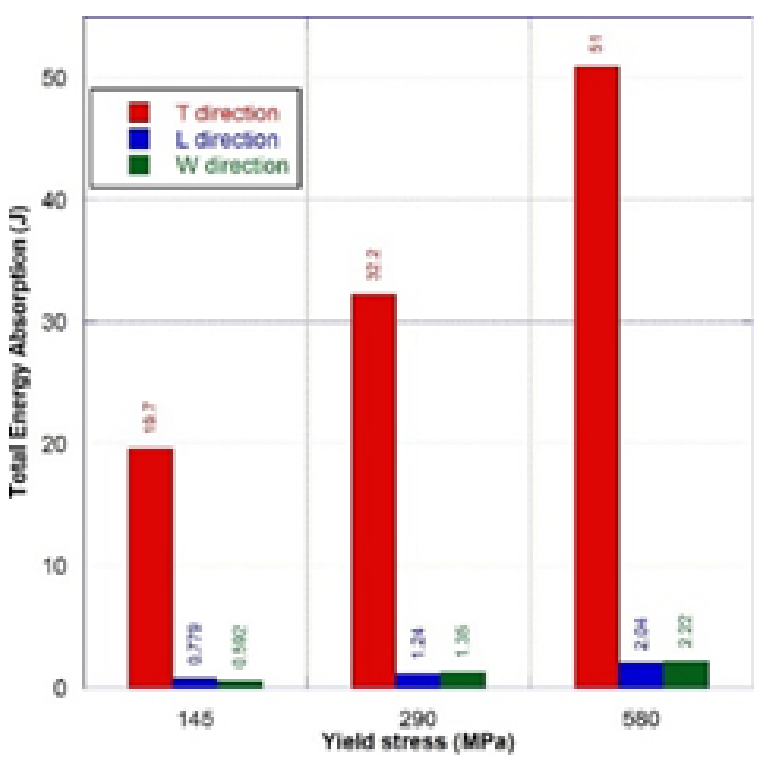

Fig. 1. Specific Energy Absorption (SEA) as a function of the cell-wall thickness direction [6]

A few different methods of honeycombs compression testing were showed by Ashab [2]. In addition to the standard method, tests of impact on perforated plates (examination of closed air influence) and smaller than honeycombs area plates (checking border effect influence) were performed. It turned out that these conditions are insignificant in static tests.

Wierzbicki [13] reported that, the plateau stress depends on the foil material and geometrical properties of analyzed structure. $\mathrm{He}$ also proposed analytical model based on stress equation. Wierzbicki achieved good agreement of the core's plateau stress values obtained by analytical and experimental investigations. His formula was successfully used by many scientists dealing with the phenomenon of progressive honeycombs crushing. All of them have achieved good agreement of results with the experimental data $[2,4,8,15]$.

Zarei Mahmoudabadi [15] investigated metallic honeycomb structures loaded with out-of-plane compression force. Furthermore, Mahmoudabadi reported theoretical analyses of plateau stress of honeycombs under crushing. He used Wierzbicki equation and new formula. Mahmoudabadi approach was a modification of Wierzbicki model by adding the curvature effects of the cylindrical wall surfaces. Mahmoudabadi reported that although the results were more accurate calculations were much more complicated.
Khoshravan and Pour [8] and Deqiang [3] showed comparison between Wierzbicki model and original experimental and numerical results. They performed Finite Element Method simulations using two approaches to modelling the structure. First was modelling full core geometry, whereas second was utilizing only one, basic, Y-shaped part of the honeycomb. Results of the investigations showed, that Y-shaped element analysis are accurate and cost effective to predict ultimate and plateau force that are comparable to experimental and theoretical data. Similar study was presented by Wilbert et al. [14] They presented experimental and numerical investigation, where FEM models consisted of 2, 4 and 16 basic unit cells. Wilbert et al. [14] reported that with the increase number of basic units they obtained smoother stress-strain curve. However, both the shape and plateau and ultimate force values remained unchanged. Authors pointed out that using smaller number of elements significantly reduced computational time.

\section{THEORETICAL STUDY}

For the purpose of presented study a theoretical approach for predicting plateau stress value was used. Basing on literature review, analytical method developed by Wierzbicki [13] was implemented. The method is based on using energy criterion. It allows calculation of crushing force value on the basis of the geometric dimensions and yield stress of core material. Wierzbicki used a perfectly plastic material model and core geometry reduced to a basic cell. He utilized the fact, that honeycomb is a symmetrical structure. It can be divided into basic elements within the uniform

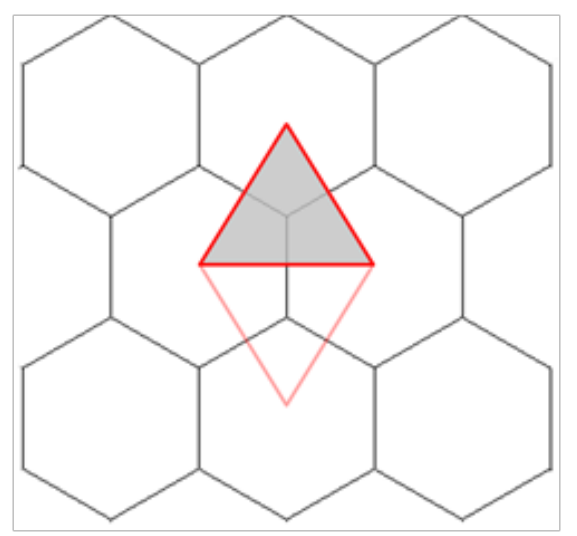

Fig. 2. Unit cell of a honeycomb structure with triangular prism 
triangular prism as shown in Fig. 2. The prism walls are symmetry planes located in the middle of each cell's wall. Part of core inside the prism is a basic Y element. Wierzbicki assumed, that a single wave contained in the basic element has a buckling factor of $\mu=0.5$, which corresponds to fully clamped boundary condition. Wierzbicki model can be only used to calculate main crushing force (area shown in Fig. 6c.). The model skips elastic response of the initially compressed structure (area shown in Fig. 6a.). and latter core densification process (area shown in Fig. 6d.).

In his study, Wierzbicki determined that the ultimate force needed to fail the $\mathrm{Y}$ shaped element forming a base cell can be calculated using equation [15]:

$$
F_{m}=8.61 \sigma_{0} t^{5 / 3} S^{1 / 3}
$$

Assuming that the base cell bound area unit has an area of:

$$
A=\frac{\sqrt{3}}{4} S^{2}
$$

the value ofplateau stress crushing the core is calculated as:

$$
\sigma_{p l}=16,56 \sigma_{0}\left(\frac{t_{c}}{S}\right)^{5 / 3}
$$

where $t_{c}$ is the wall foil thickness, $S$ is cell size and $\sigma_{0}$ is yeld stress of core foil material. Dimensions of basic core cell element used in analyses are shown on Fig. 3. As can be seen in the picture, two parts of basic $\mathrm{Y}$ element have single thickness while common part (where core foils are connected) have double thickness.

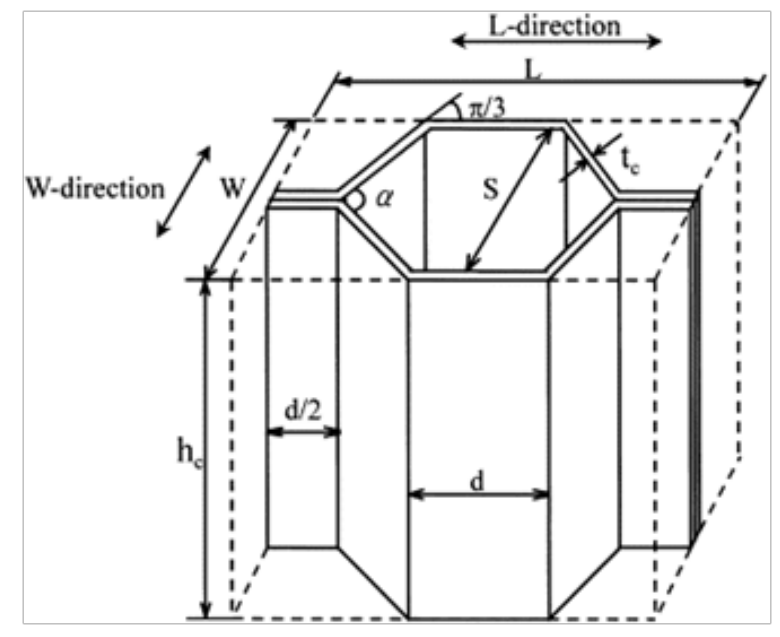

Fig. 3. Geometry of basic cell core elements [15]

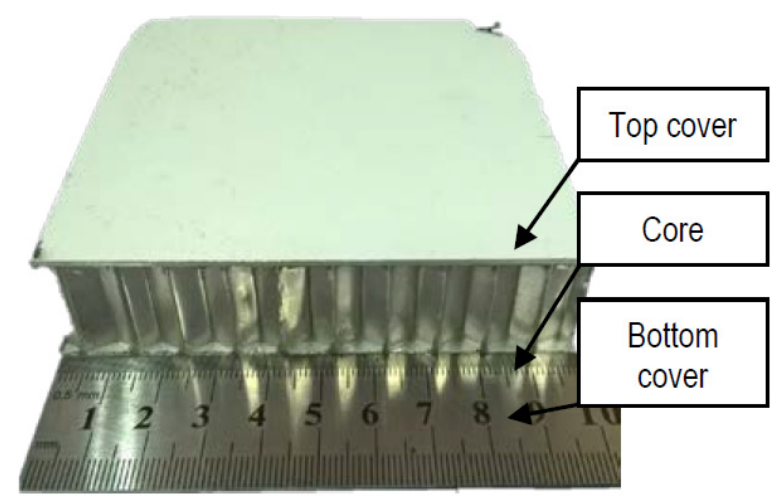

Fig. 4. Double skinned honeycomb used to prepare specimens

\section{EXPERIMENTAL INVESTIGATION}

Prior to numerical simulation, experimental out-of-plane compression tests were carried out for the purpose of model validation. Test procedure for determining plateau stress was based on methodology described in MIL STD 401B [11].

Investigations were performed for commercially available ALUBOND ${ }^{\circledR}$ Alucore material. It is a sandwich structure completely made of aluminum. Thin-walled honeycomb core is bonded by glue adhesion to covers (skins). Sandwich plate is shown in Fig. 4. To maintain correspondence with theoretical model, the material was examined to obtained its geometrical features. Basic core characteristics measured according to Fig. 3. are: $t_{c}=0,09 \mathrm{~mm}, \mathrm{~S}=9,5 \mathrm{~mm}$, $\mathrm{H}=23 \mathrm{~mm}$. Specimen prepared for tests had dimensions 60x60 mm.

Both core and covers were made of aluminum alloy. Top and bottom covers had a thickness of $1.0 \mathrm{~mm}$ each. Mechanical properties of Al 3105H25 aluminum are shown in the Tab. 1.

Aim of the investigation was to measure force and displacement in out-of-plane compression of the analyzed structure. Experiments were per-

Table 1. Mechanical properties of A1 3105-H25 [9]

\begin{tabular}{|c|c|c|c|}
\hline Parameter & Symbol & Unit & Value \\
\hline Modulus of Elasticity & $\mathrm{E}$ & $\mathrm{MPa}$ & 69 \\
\hline Shear Modulus & $\mathrm{G}$ & $\mathrm{MPa}$ & 25 \\
\hline Tensile Strength, Yield & $\sigma_{0}$ & $\mathrm{MPa}$ & 160 \\
\hline $\begin{array}{c}\text { Tensile Strength, } \\
\text { Ultimate }\end{array}$ & $\sigma_{\mathrm{u}}$ & $\mathrm{MPa}$ & 180 \\
\hline Shear Strength & $\mathrm{T}_{\mathrm{u}}$ & $\mathrm{MPa}$ & 105 \\
\hline Elongation at Break & $\varepsilon_{\mathrm{u}}$ & $\%$ & 8 \\
\hline
\end{tabular}




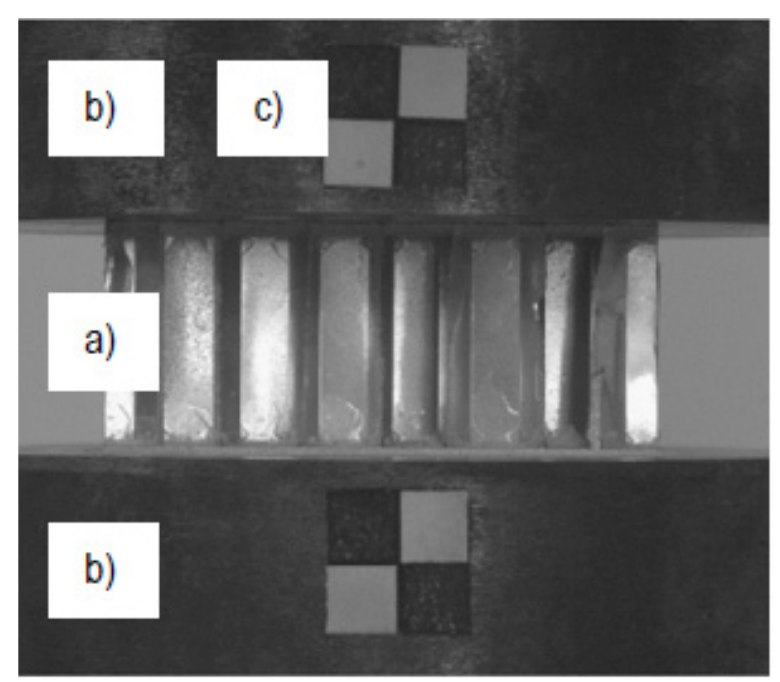

Fig. 5. Experimental setup: a) sample, b) steel plate, c) high speed camera marker

formed using INSTRON 8802 universal fatigue rated servohydraulic testing system. Specimen during test is shown in Fig. 5. As can be seen in the picture, tests were performed using circular steel plates (b) between which the sample (a) was placed. This allowed the uniform distribution of force applied to the specimen surface. For the purpose of filming, markers were added to allow tracing displacement of circular plates using video data analysis software. Test was performed at the bottom head speed of $\mathrm{v}=4 \mathrm{~mm} / \mathrm{s}$.

During tests, displacement of the machine's piston and the reaction force in the direction normal to the sample midsurface were recorded. Data from the test system was registered using ESAM Traveler bridge. A recording frequency of $0.01 \mathrm{~s}$ was used. In addition to force-displacement acquisition, movies were recorded using Phantom V12 high speed camera with frame rate of 25 fps.

Axial force versus machine piston displacement values were obtained as a result of the trials. For each test, stress and volumetric strain values were calculated using each specimen area. Volumetric strain is often used when cellular materials are concerned. It allows better representation of structure response to load, as honeycombs deform and fail globally. Stress - volumetric strain curves obtained as a result of each test are shown in Fig. 6. A total number of 5 tests were performed. It can be observed, that the results are repeatable. Furthermore, obtained data for each tests are in good agreement with numbers reported in literaturę [1, $6,7,10,12,13]$ for aluminum honeycomb struc- tures. One can observe characteristic regions in which different phenomena occurs. The data was then processed in order to obtain maximum and plateau stress values. For analyzed structure mean plateau stress was $1,75 \mathrm{MPa}$, whereas mean maximum stress value reached 4,25 $\mathrm{MPa}$.

As shown in Fig. 6., it is possible to distinct four regions of plot corresponding to physical honeycomb specimen behavior. First of them is an elastic region, where structure behavior is quasi-linear. Maximum recorded stress value was 4.25 MPa. When critical force was reached, thin walls of the structure began to collapse in in plane directions, which corresponds to stress value drop to $1.2 \mathrm{MPa}$. It is depicted as region b in Fig. 6. When first wave on core walls is formulated, stress value started to increase. Maximum value is the end of region $b$. Later on, in region $c$, more waves were formed and the force value remained constant. This force value is called the plateau stress. The plateau area is very characteristic of honeycomb structures during out-of-plane compression. In this region, most of impact energy is absorbed. When walls began to contact with each other, there was a rapid increase of stress observed. The region is depicted as area $\mathrm{d}$. In this region, specimen material is densified. Ultimately, for fully compressed material maximum stress value was obtained.

Basing on presented results mean values of plateau and maximum stress were obtained. Using mathematical formulas, a confidence intervals were also calculated:

$\sigma_{\max }=4.25 \mathrm{MPa}, 95 \%$ confidence interval $<4.18: 4.33>$, $\sigma_{\text {plateau }}=1.75 \mathrm{MPa}, 95 \%$ confidence interval
$<1.69: 1.81>$.

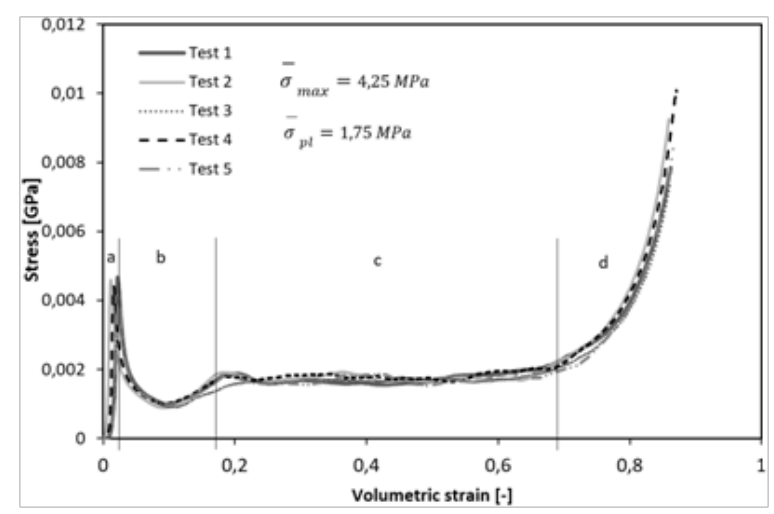

Fig. 6. Stress - vol. strain curves, experimental data; a) elastic region, b) buckling collapse region, c) plateau stress region, d) densification region 
In latter analyses the data for each test shown in Fig. 6. was averaged in order to compare mean values with simulation results.

\section{NUMERICAL MODELLING}

\section{Numerical model description}

Next part of the study was numerical analysis of honeycomb structure under out-of-plane compression. The analyses were performed using two approaches. One of evaluated methods was modelling entire core geometry. Second approach was based on basic $\mathrm{Y}$ element as proposed by Wierzbicki [13]. In presented study these two methods are compared. Literature review showed, that modelling behavior of honeycomb structures can be accurately done using Finite Element Method [3, 8, 14]. The FE method was chosen to perform analyses.

Validation of presented study was based on quasi-static experimental tests where force was a function of displacement. Due to quasi-static character of experimental validation trials, implicit time integration scheme was chosen. As well as that, modelled structure had geometry features leading to complex behavior under load. Examined specimen were made of A1 3105-H25 aluminum, which shows strain-rate dependent behavior. Due to those factors, for the purpose of numerical study a non-linear finite element method implemented in LS-DYNA software was used.

First of the presented approaches was modelling the whole geometry of honeycomb structure. This method allowed observing global behavior of entire structure geometry response to crushing load. For this purpose, the geometrical model of specimen was created. Due to expected deformation of geometry and distortion of elements, fully integrated shells were chosen. Mesh density used for calculation was $0.4 \mathrm{~mm}$. The model is depicted in Fig. 6. Analyzed structure consisted of foil cell halves bonded to each other at common planes. These common planes are depicted as blue walls in Fig. 7. These walls had double foil thickness $(t=2 T)$ whereas remaining walls, depicted as red in Fig. 7. had a single thickness ( $t$ $=1 \mathrm{~T}$ ). Adhesive connection between walls was not simulated in this model. Instead, elements on common walls had their thickness equal to double of basic foil thickness assuming that double wall acts like a single thick part. Nodes located on the top of each honeycomb walls were fully clamped. This boundary condition was used for calculation of $\mathrm{Z}$ axis reaction force value. In the bottom of

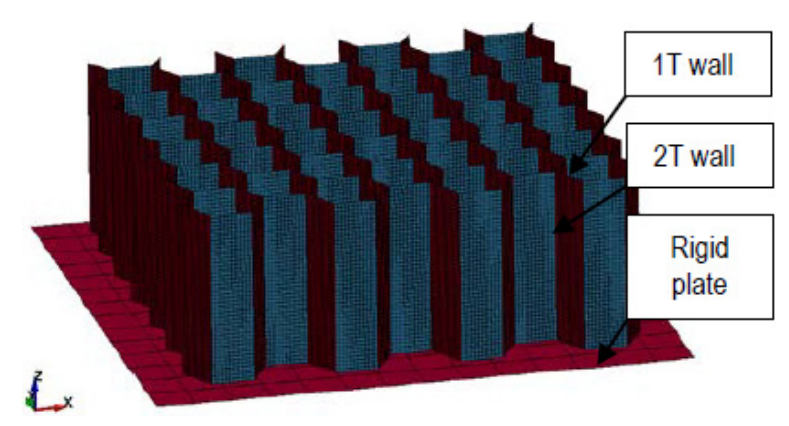

Fig. 7. Geometrical representation of honeycomb numerical mode

the core, there was also rigid plate moving in its normal direction upwards. Core nodes on bottom surface had possibility of movement only in Zaxis. These boundary conditions simulated the connection between core and aluminum faces, which was not modelled. The rigid plate moved from initial position up to $22 \mathrm{~mm}$ in Z-axis.

To simulate behavior of Al 3105-H25 material, elastic plastic constitutive relation was used. In LS-DYNA the model is implemented as MAT_003_PLASTIC_KINEMATIC (Hallquist, 2006). This is a bilinear stress-strain model recommended for isotropic materials with optional kinematic hardening. The model can be used for simulation of strain-rate dependent materials using Cowper-Symonds constants. Literature reports, that such approximation of material behavior is sufficient for the purpose of modelling aluminum during honeycomb crushing [3, 8, 14]. For the purpose of presented study no work hardening or rate effects were considered. Material properties of aluminum are shown in Tab. 1.

Second approach for modelling the behavior of honeycomb structure utilized the semi-analytical model proposed by Wierzbicki [13]. The idea was to replace full geometry of the core by single, basic Y-shaped cell shown in Fig. 8. Such simplification of geometry allows for dense mesh

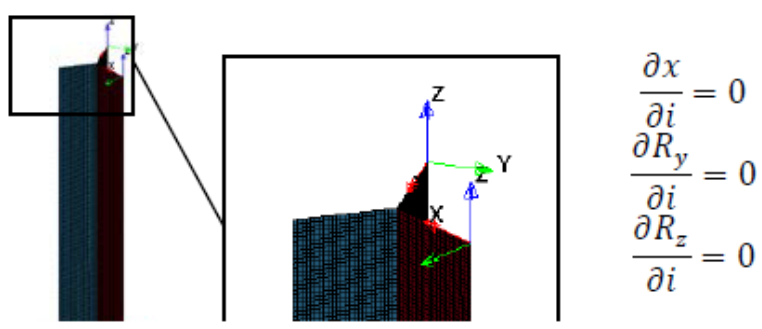

Fig. 8. Y-shaped basic element with boundary conditions 
of component. Therefore, it is possible to obtain better accuracy during observation of progressive failure process. However, for this kind of analyses, there is a need for additional boundary conditions and local coordinate systems for stating them. In Fig. 8 there are visible local axes of coordinate systems on red walls. Corresponding boundary conditions are also given.

\section{Analysis of results}

After performing numerical analysis of presented models, the results were evaluated using experimental data. Values of $\mathrm{Z}$-axis reaction force and deformation images were recorded. Fig. 9. shows comparison between the results of simulation with pictures obtained during tests for selected subsequent crushing states. Progressive failure of core walls is visible. Comparing the results, it can be observed, that both the model and specimen behaved similarly.

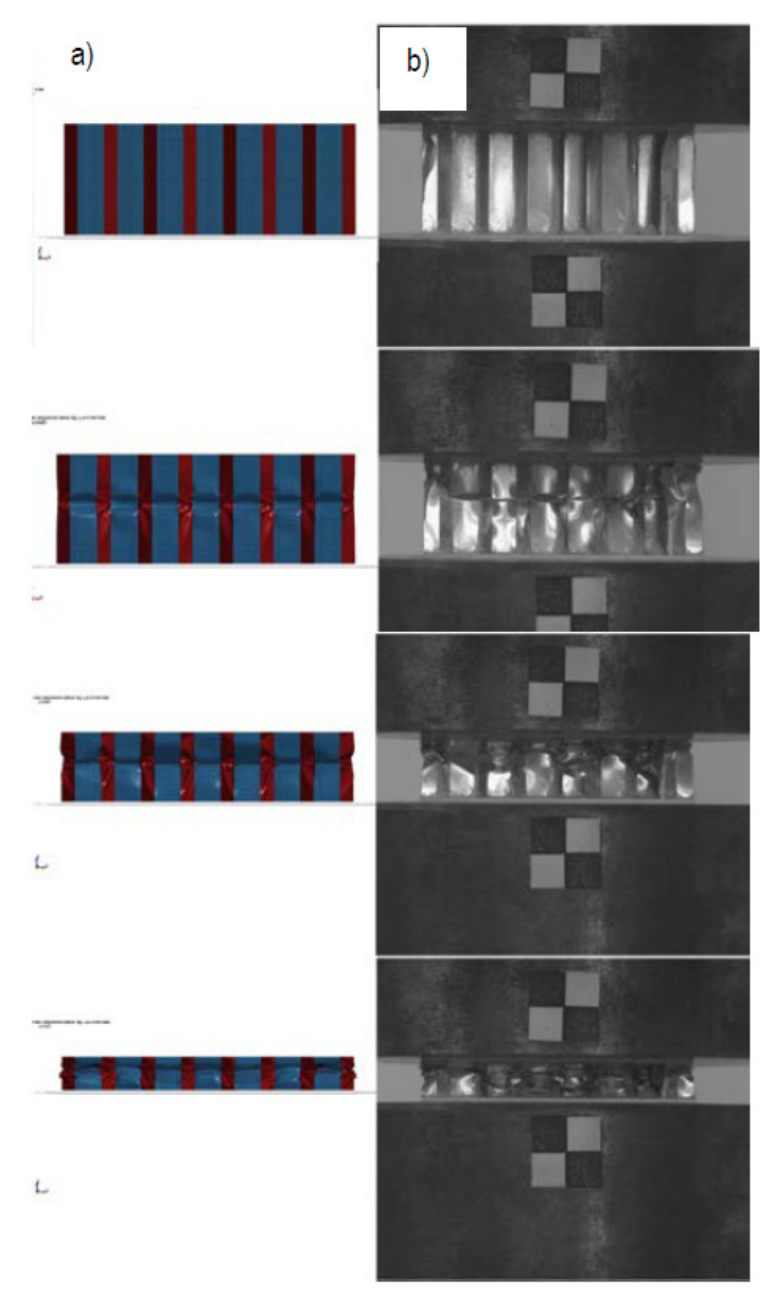

Fig. 9. Deformation of honeycomb in selected moments of the test: a) FEM analysis, b) experiment
Fig. 10. presents selected moments of progressive crushing of honeycomb core structure. In subsequent pictures there are visible: a) undeformed structure, b) initialization of buckling, c) structure crushing when plateau stress in observed and d) beginning of core densification. Comparing simulation with specimen tests, it is visible that, failure process observed in experimental investigation was less predictable. It is caused by idealizations in numerical model. Despite that, good agreement in deformation modes and number of waves on single core's wall was observed. In both analyses, the buckling initiation took place in the specimen height center. Until the newly created walls had not come into contact, further waves did not form. Edges, which were jointed adhesively to the skins were not bended until the end of the test.

Deformation process showed in Fig. 10. can be divided into intervals, same as in full geometry model. At first, elastic compression (a) can be observed. Next part is stability loss and beginning of the buckling process (b-c). Finally, after collapsing, the structure was fully compacted (d). Shape and number of waves on every wall depended on core height and mesh density. Both simulations showed, that the mechanism of core crushing after stability loss is local bending and contact between bent walls along their common edge. First wave shape corresponds to first buck- a)

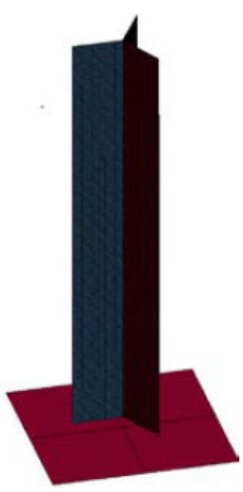

c)

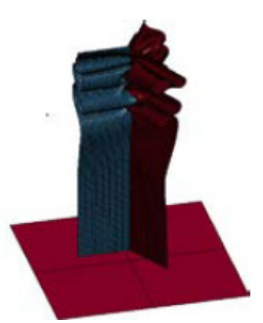

b)

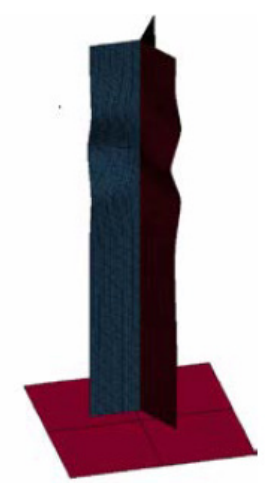

d)

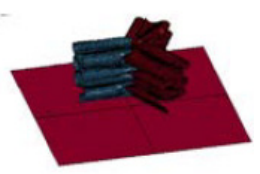

Fig. 10. Progressive crushing of Y-shaped element in selected moments: a) $0 \mathrm{~s} \mathrm{~b}$ ) $0,2 \mathrm{~s} \mathrm{c)} 5,5 \mathrm{~s}$ d) $10 \mathrm{~s}$ 


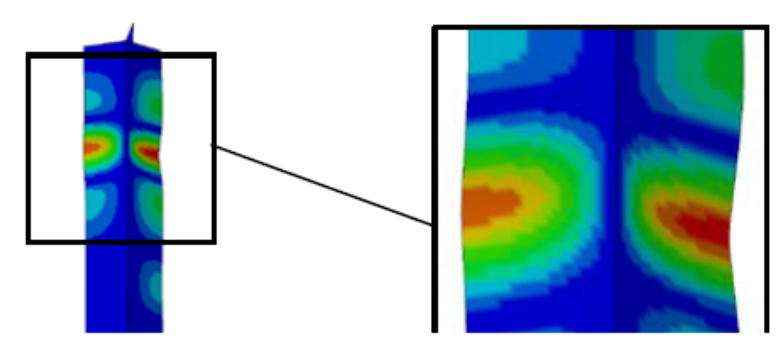

Fig. 11. Beginning of buckling process

ling mode with wavelength equal to $1 / 3$ cell size as it is shown on Fig. 11.

As a result of analyses, stress - volumetric strain plots were obtained. Curves obtained in the experiment and both types of numerical analysis were summarized in one graph. They were also compared with the outcome of calculations using Wierzbicki equation. All of resultant curves are shown in Fig. 12.

Plots obtained using different methods are similar. As visible in Fig. 12., elastic modulus and critical stress value for both numerical models were higher than the experimental ones. This is due to lack of imperfections in numerical model, leading to more stiff structure response. The moment of stability loss is also different. This is a result of measuring piston vertical displacement directly from the testing machine instead of using extensometer. Plateau stress phase was similar for all applied methods. The force course achieved in Y-shaped element was less smooth than in full geometry model. As well as that, beginning of densification was observed at higher strain value for Y-shaped element. This is related to fewer walls leaning against each other and thus increasing the reaction force. All results

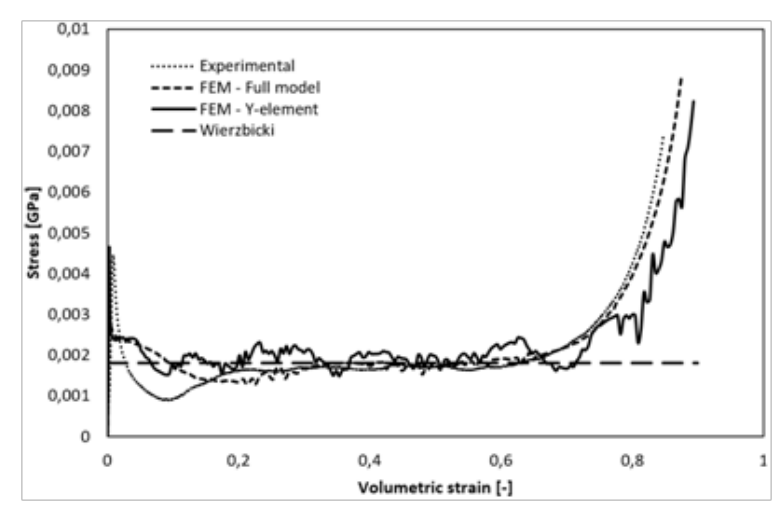

Fig. 12. Comparison of stress - vol. strain curves obtained by different methods in plateau part overlap with the theoretical value determined using Wierzbicki equation [13].

\section{PARAMETRICAL ANALYSES}

Last part of the study were parametric analyses made on sandwich structure with honeycomb core. Basic geometrical parameters characterizing the material were $S=9,5 \mathrm{~mm}$ and $t_{c}=0,09$ $\mathrm{mm}$. Aim of the investigation was to check the influence of foil thickness and cell size on resultant plateau stress value. In order to investigate the influence of geometric characteristics several further analyses with only one parameter under consideration were conducted. Range of parameters tested in each case is shown in the Tab. 2.

At first, the influence of wall thickness was analyzed. Four thicknesses were tested: $\mathrm{t}_{\mathrm{c}}=0,02 \mathrm{~mm}$, $\mathrm{t}_{\mathrm{c}}=0,05 \mathrm{~mm}, \mathrm{t}_{\mathrm{c}}=0,12 \mathrm{~mm}$, and compared with basic $t_{c}=0,09 \mathrm{~mm}$. Deformation images and stress - volumetric strain curves were obtained. Stress value in the plateau range were averaged. Their values were later compared. Fig. 13 presents images of deformed structure at the moment when they lost stability. It can be observed, that height of the bending initiation point increases with the increase of wall thickness. As well as that, the number of rising waves decreases and their length increases.

Table 2. Geometric parameters of honeycomb studied in analyses

\begin{tabular}{|c|c|c|c|}
\hline Parameter & Symbol & Unit & Value \\
\hline \multirow{3}{*}{ Wall thickness } & \multirow{3}{*}{$t_{c}$} & $\mathrm{~mm}$ & 0,02 \\
\hline & & $\mathrm{mm}$ & 0,05 \\
\hline & & $\mathrm{mm}$ & 0,12 \\
\hline \multirow{3}{*}{ Cell size } & \multirow{3}{*}{ S } & $\mathrm{mm}$ & 7 \\
\hline & & $\mathrm{mm}$ & 5,2 \\
\hline & & $\mathrm{mm}$ & 3,2 \\
\hline
\end{tabular}

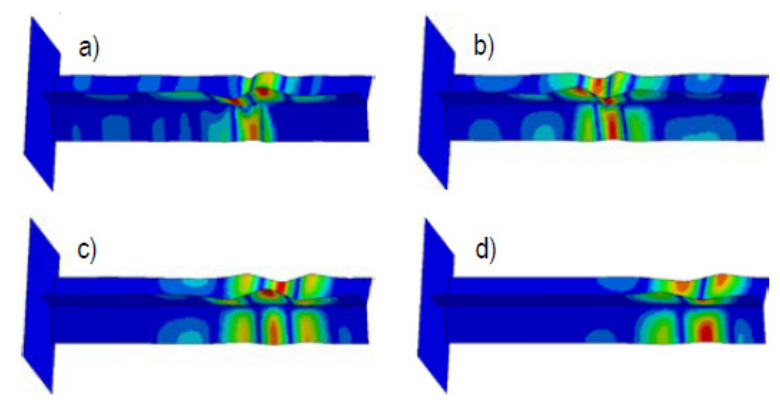

Fig. 13. Moment of initiation buckling process in different wall thickness structures: a) $\left.t_{c}=0,02 \mathrm{~mm}, \mathrm{~b}\right) \mathrm{t}_{\mathrm{c}}$ $\left.=0,05 \mathrm{~mm}, \mathrm{c}) \mathrm{t}_{\mathrm{c}}=0,09 \mathrm{~mm}, \mathrm{~d}\right) \mathrm{t}_{\mathrm{c}}=0,12 \mathrm{~mm}$ 

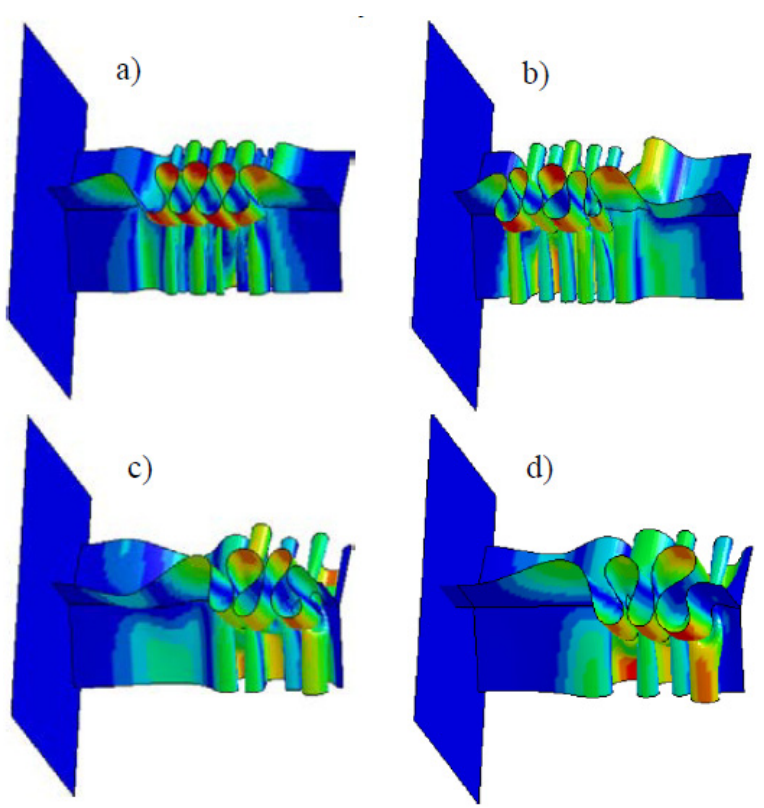

Fig. 14. Deformatied honeycomb after $50 \%$ plane displacement: a) $\left.\left.\mathrm{t}_{\mathrm{c}}=0,02 \mathrm{~mm}, \mathrm{~b}\right) \mathrm{t}_{\mathrm{c}}=0,05 \mathrm{~mm}, \mathrm{c}\right) \mathrm{t}_{\mathrm{c}}=$ $0,09 \mathrm{~mm}, \mathrm{~d}) \mathrm{t}_{\mathrm{c}}=0,12 \mathrm{~mm}$

It was noticed that crushing structure with thicker walls resulted in less number of observed waves. This effect is shown in Fig. 14. In each of the analyzed cases, the mechanism of the core destruction was the same. Differences were observed in their shape. Each new wave began to emerge after closure of previous one. With the increase of foil thickness the bending radius increased as well. Therefore, total number of waves was lower. In all analyzed cases edges on the top and bottom planes were undeformed. This was due to assumed boundary conditions.

Second part of parametric study was the investigation of cell size influence on plateau stress. Four values of cell size were selected for analysis: basic size $\mathrm{S}=9,5 \mathrm{~mm}, \mathrm{~S}=7 \mathrm{~mm}, \mathrm{~S}=5,2 \mathrm{~mm}$

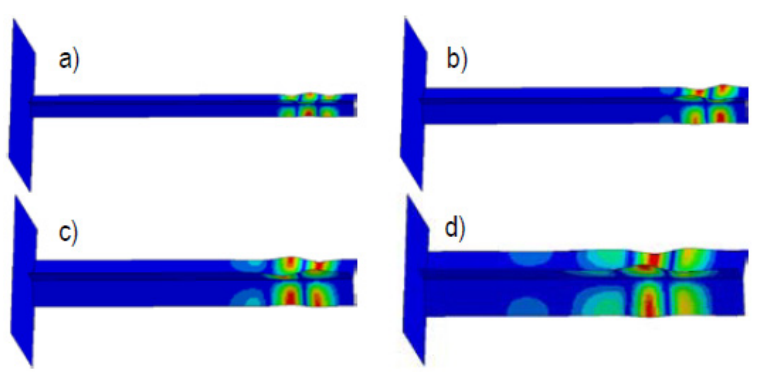

Fig. 15. Moment of initiation buckling process in different cell size structures: a) $\mathrm{S}=3,2 \mathrm{~mm}$, b) $\mathrm{S}=5,2$ $\mathrm{mm}, \mathrm{c}) \mathrm{S}=7 \mathrm{~mm}$, d) $\mathrm{S}=9,5 \mathrm{~mm}$

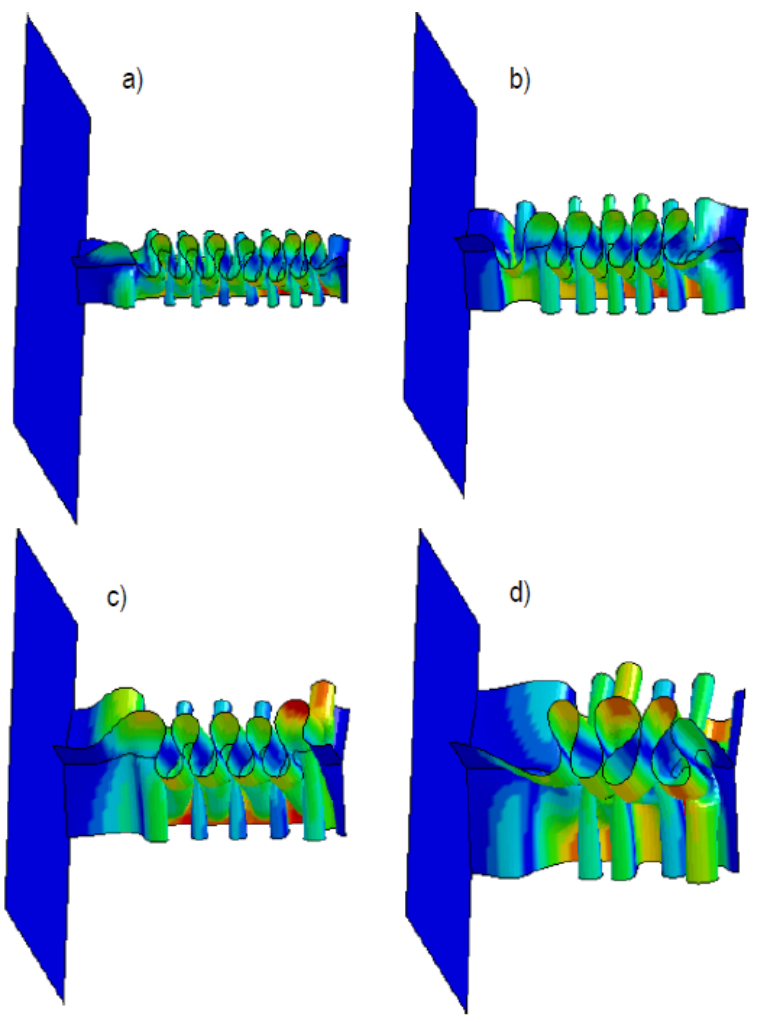

Fig. 16. Deformatied honeycomb after $50 \%$ plane displacement: a) $\mathrm{S}=3,2 \mathrm{~mm}$, b) $\mathrm{S}=5,2 \mathrm{~mm}, \mathrm{c}) \mathrm{S}=7$ $\mathrm{mm}$, d) $\mathrm{S}=9,5 \mathrm{~mm}$

and $\mathrm{S}=3,2 \mathrm{~mm}$. All tests were conducted under the same conditions. Each Y-shaped element had the same nodes numbers on the wall along core material height. In Fig. 15 there are presented moments of the initiation of deformation for analyzed cell sizes. In all cases observed buckling mode was the same. As well as that, the collapse process began near the top surface. In each case the wavelength was equal to $1 / 3$ of cell size.

The study of cell size influence showed, that cells deform evenly along their length. Fig. 16. shows that with increasing cell size fewer and longer waves were observed. On the other hand, larger number of waves over the wall length caused stiffness increase due to presence of more bending points.

Averaged plateau stress values for numerical tests concerning foil thickness and cell size were compared with the results of theoretical calculations made with Wierzbicki equation [13]. As shown in Fig. 17., plateau stress value increased exponentially with wall thickness. It is an effect of higher wall stiffness against bending forces after stability loss for thicker walls. Theoretical values and simulation re- 


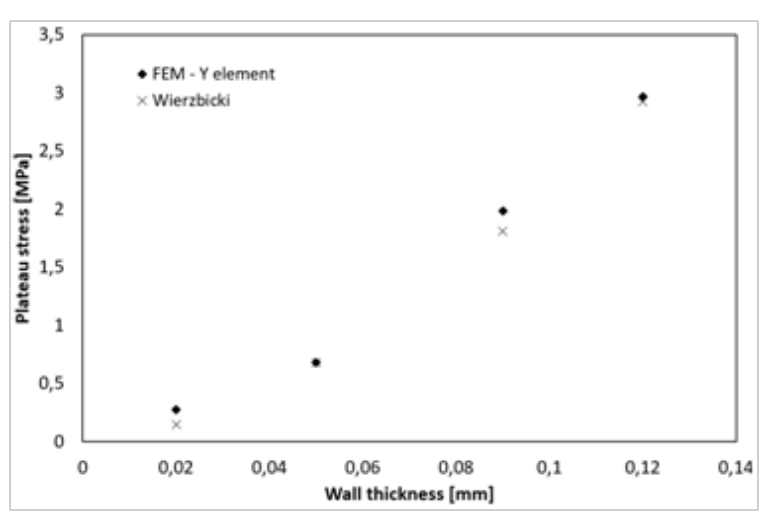

Fig. 17. Plateau stress value versus wall thickness for Y-shaped element

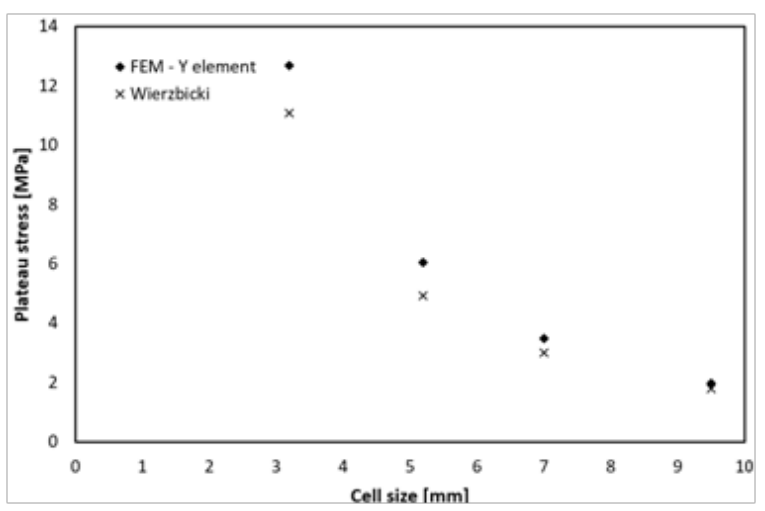

Fig. 18. Plateau stress value versus cell size Y-shaped element

sults are in good agreement. The reverse trend was observed with rising cell size. Parametric study results are depicted in Fig. 18. Larger size resulted in decrease of global structure stiffness. This is due to lower number of waves along the wall and therefore, less bending points and interlocking walls fragments.

\section{CONCLUSIONS}

The fundamental problem in energy-absorbing honeycomb structures design is to assess the plateau stress value. This value allows estimating the amount of kinetic energy, that can be dissipated. This paper presented four approaches that can be used for this purpose.

Theoretical approach provides limited data, as only plateau stress can be calculated. On the other hand, experimental technique gives all material characteristics but is the most expensive because it involves testing a large number of specimen. Costs can be reduced by performing numerical simulations. Time needed for performing calculation can be further reduced by using a Y-shaped element. Analysis showed, that this approach gave reliable results with minor differences compared to theoretical and experimental values. This conclusion is consistent with observations made by Wilbert et al. [14].

Experimental out-of-plane compression tests of ALUCORE honeycomb proved, that the examined material has energy-absorbing capabilities. During study, analyzed structure mean plateau stress was 1,75 MPa whereas mean maximum stress value reached 5,25 MPa. The behavior of material was in good agreement with numbers reported in literaturę $[6,10,12]$.

The study showed that the initial and final deformation mode strongly depends on the geometric parameters of the honeycomb structure. The forces required to crush the core grew along with the increase in wall thickness, and decrease with the cell size. To design energyabsorbing structures it is recommended to carry out theoretical calculations with the Wierzbicki formula or its modifications, and to perform analyses using a Y-shaped element. This method is superior to experimental tests and full structure analysis.

\section{Acknowledgements}

The work has been accomplished under the research project No. DOB-BiO/001/05/2014 financed by the National Centre for Research and Development.

\section{REFERENCES}

1. Ashab, A., Ruan, D., Lu, G., \& Wong, Y. C. Quasistatic and dynamic experiments of aluminum honeycombs under combined compression-shear loading, Materials \& Design, 97, 183-194, 2016.

2. Ashab, A., Ruan, D., Lu, G., Xu, S., \& Wen, C. Experimental investigation of the mechanical behavior of aluminum honeycombs under quasi-static and dynamic indentation, Materials \& Design, 74, 138-149, 2015.

3. Deqiang, S., Weihong, Z., \& Yanbin, W. Mean outof-plane dynamic plateau stresses of hexagonal honeycomb cores under impact loadings, Composite Structures, 92(11), 2609-2621, 2010.

4. Gibson, L. J., \& Ashby, M. F. Cellular solids : structure and properties, Cambridge University Press, 1999.

5. Hallquist, J. LS-DYNA ${ }^{\circledR}$ theory manualLivermore Software Technology Corporation, 2006. 
6. Ivañez, I., Fernandez-Cañadas, L. M., \& SanchezSaez, S. Compressive deformation and energy-absorption capability of aluminium honeycomb core, Composite Structures, 2017.

7. Kee Paik, J., Thayamballi, A. K., \& Sung Kim, G. The strength characteristics of aluminum honeycomb sandwich panels, Thin-Walled Structures, 35(3), 205-231, 1999.

8. Khoshravan, M. R., \& Najafi Pour, M. Numerical and experimental analyses of the effect of different geometrical modelings on predicting compressive strength of honeycomb core, Thin-Walled Structures, 84, 423-431, 2014.

9. Matweb - Your Source for Materials Information, MatWeb, 1-2, 2015.

10. McFarland, R. The development of metal honeycomb energy-absorbing elements. Technical report no. 32-639, California, 1964.
11. MIL-STD-401B. Sandwich Constructions and Core Materials, General Test Methods, 1967.

12. Wang, Z., Liu, J., \& Hui, D., Mechanical behaviors of inclined cell honeycomb structure subjected to compression, Composites Part B: Engineering, 110, 307-314, 2017.

13. Wierzbicki, T. Crushing analysis of metal honeycombs, International Journal of Impact Engineering, 1(2), 157-174, 1983.

14. Wilbert, A., Jang, W.-Y., Kyriakides, S., \& Floccari, J. F. Buckling and progressive crushing of laterally loaded honeycomb, International Journal of Solids and Structures, 48(5), 803-816, 2011.

15. Zarei Mahmoudabadi, M., \& Sadighi, M. A theoretical and experimental study on metal hexagonal honeycomb crushing under quasi-static and low velocity impact loading, Materials Science and Engineering: A, 528(15), 4958-4966, 2011. 